\title{
HYPERMETABOLISM ON PEDIATRIC POSITRON EMISSION TOMOGRAPHY SCANS OF BRAIN GLUCOSE METABOLISM: What does it signify?
}

\author{
Harry T. Chugani, MD \\ Department of Neurology \\ NYU Langone School of Medicine \\ New York, NY
}

\section{Correspondence:}

Harry T. Chugani, MD

NYU Langone Comprehensive Epilepsy Center

223 East $34^{\text {th }}$ Street

New York, NY 10016, USA

Email: Harry.Chugani@nyulangone.org

ORCHID 0000-0001-5080-2106 


\begin{abstract}
When interpreting clinical 2-deoxy-2[18 F]fluoro-D-glucose (18F-FDG) positron emission tomography (PET) scans of the brain (excluding tumors) in children, the typical abnormality is hypometabolism of various brain regions. Focal areas of hypermetabolism are noted occasionally and the usual interpretation is that the hypermetabolic region represents a seizure focus. In this review, we discuss and illustrate the multiple causes of hypermetabolism that should not be interpreted as seizure activity could potentially lead to an incorrect interpretation of 18F-FDG PET studies. Various causes or conditions where focal hypermetabolism can be encountered on 18F-FDG PET studies include interictal hypermetabolism, Sturge-Weber syndrome, changes associated with brain plasticity following injury, Rett syndrome, hypoxicischemic brain injury, various inborn errors of metabolism, and autoimmune encephalitis. The radiologist or nuclear medicine physician interpreting clinical 18F-FDG PET studies should be aware of these circumstances to provide accurate assessment of the study.
\end{abstract}

Key words: 18F-FDG PET, brain, hypermetabolism, epilepsy

Disclosure: No potential conflicts of interest relevant to this article exist. 
Positron emission tomography (PET) scans of glucose metabolism using the radiotracer 2-deoxy-2 $\left[{ }^{18} \mathrm{~F}\right]$ fluoro-D-glucose (18F-FDG) have been performed for over 3 decades and have been incorporated into clinical practice, particularly in oncology. In adults, brain 18F-FDG PET scans are approved for the evaluation of dementia, intractable epilepsy, and to differentiate between recurrent brain tumor and radiation necrosis. In children, however, brain 18F-FDG PET scans are approved in the evaluation of intractable epilepsy for potential epilepsy surgery and for brain tumors. We have recently presented data and made the argument that 18F-FDG PET scans are also clinically valuable in the management of children with neurodegenerative disorders and have recommended that approval should be extended to include this population as well (1).

In the interpretation of 18F-FDG PET scans, the typical abnormality is hypometabolism of various brain regions. For clinical studies, hypometabolic brain regions are determined on visual inspection by comparison with adjacent regions and to the contralateral hemisphere. Various semiquantitative objective methods are also used in some centers to augment visual interpretation. However, brain regions showing hypermetabolism are noted occasionally and the interpretation of such images can be very challenging. The usual conclusion is that the hypermetabolic region represents an epileptic focus (if small) or an epileptogenic region (if large). While this interpretation may be correct in most patients with epilepsy, it may be incorrect in a considerable number of cases, as will be demonstrated below, and lead to erroneous interpretation of the images. It should also be pointed out that the semiquantitative objective methods designed to aid in visual analysis have been validated to detect only hypometabolism and not hypermetabolism. In this review, which is focused mainly on children, we discuss and illustrate the multiple causes of hypermetabolism that can be encountered on 18F-FDG PET studies based on a review of previously published studies. A discussion of the hypermetabolism seen in malignant brain tumors is not within the scope of this review. Since this is a review of previously published studies, institutional review board approval was not required.

\section{F-FDG PET HYPERMETABOLISM IN EPILEPSY}

Unlike with single photon emission computed tomography (SPECT), where ictal scans can be planned, it is not practical to schedule an ictal PET scan because of the short half-life of 18F (about 110 minutes). Patients (particularly children) with frequent seizures may occasionally experience a seizure during the approximately 30 minute 18 F-FDG uptake period, thus yielding an ictal PET scan. In some institutions, patients with frequent seizures are monitored with an electroencephalogram during the tracer uptake period and, if a clinical or subclinical seizure occurs, its localization, timing and duration can be accurately analyzed (2). If the seizure occurs early enough (approximately first 10 minutes) during the tracer uptake period and lasts for more than a few minutes, hypermetabolism may be seen on the images and may provide useful localization data (Figure 1A), both from the PET scan itself and from the concurrent electroencephalogram if performed. Brief seizures or seizures occurring late in the relatively long 18 F-FDG uptake period will usually not show hypermetabolism. 
Less well known and often missed is the phenomenon of 'interictal hypermetabolism' $(2,3)$. This occurs when the patient's electroencephalogram shows a focal interictal epileptiform discharge that persists throughout much of the tracer uptake period. No actual seizure is recorded clinically or on the electroencephalogram, yet the PET images show focal hypermetabolism in a cortical region (Figure 1B) corresponding to the focal discharge seen on the electroencephalogram. This phenomenon is similar to observations from experimental animal models of focal epilepsy induced by electrical or chemical kindling, and 14C-2deoxyglucose autoradiography reveals hypermetabolism in the spiking focus without an actual clinical seizure recorded on the electroencephalogram or a clinical seizure observed in the animal (4). The high energy demand of the persistent epileptic spiking is sufficient to produce an observable hypermetabolism in the 14C-2-deoxyglucose experimental model, as well as with 18F-FDG in human PET studies. Therefore, patients known to have actively spiking foci who are undergoing an 18F-FDG PET study should be monitored with electroencephalography during the uptake period in order to allow an accurate interpretation of the scan, a practice only rarely followed in clinical PET centers. Interictal hypermetabolism is more commonly seen in children than in adults presumably because the electroencephalograms in epileptic children often show more robust epileptiform activity than in adults. When concurrent electroencephalography during PET is not feasible, a review of previous electroencephalograms in the patient may also aid in the accurate interpretation of the 18F-FDG PET study.

\section{HYPERMETABOLISM IN STURGE-WEBER SYNDROME}

In children with Sturge-Weber syndrome (SWS), the typical finding on 18F-FDG PET is hypometabolism in the involved cortex, often with extension to additional areas beyond the vascular abnormality (leptomeningeal angioma) seen on magnetic resonance imaging (MRI) $(5,6)$. However, in the early stages of the evolution in SWS, affected infants may show a paradoxical pattern of hypermetabolism interictally in the cortex underlying the leptomeningeal angioma. As the disease progresses, the hypermetabolic area becomes hypometabolic and atrophied, indicating a dynamic course of brain injury in this disorder (5). Different regions within the affected hemisphere may undergo this process independently (Figure 2A). Eventually all affected regions become atrophied and hypometabolic. This can occur in unilateral as well as bilateral cases of SWS (Figures 2B, 2C). Early hypermetabolism seen on the 18F-FDG PET scans of children with SWS is almost never the result of seizure activity unless an actual seizure is witnessed during the tracer uptake period. Moreover, the hypermetabolism in SWS is typically not due to interictal spiking since this is not a common finding in their EEGs, which more typically show focal slowing and background attenuation over the angioma.

The finding of focal glucose hypermetabolism in the early stages of SWS should not be misinterpreted as a seizure focus nor an area of ischemia requiring more glucose because it has switched to anaerobic metabolism. Indeed, these considerations were dismissed after findings reported from SPECT studies which showed hyperperfusion in affected regions in babies and infants even prior to seizures onset (7). Both the 18F-FDG PET and SPECT observations are consistent with an earlier study which found accelerated myelination in the affected hemisphere on MRI scans $(8)$. 
Glucose hypermetabolism, hyperperfusion and accelerated myelination in focal brain regions during the early course of SWS are likely interrelated phenomena indicating dynamic changes within these regions leading eventually to tissue damage. A study using magnetic resonance spectroscopy (MRS) to address the underlying cause of the hypermetabolism found mean ipsilateral/contralateral glutamate/creatine ratios to be highest in the youngest of the 10 children evaluated, with a strong positive correlation to their seizure frequency scores. All scans were performed in the interictal state as verified by concurrent EEG monitoring. In 5 children, increased glutamate concentrations in the affected hemisphere were seen in the same areas manifesting current or previous hypermetabolism on 18F-FDG PET (9). The authors suggested that increased glutamate concentrations are common in young children with SWS and likely to be associated with their tissue damage and epilepsy. These findings are also consistent with observations from a previous study suggesting that the early cortical hypermetabolism may be predictive of subsequent severe epilepsy (10).

Another phenomenon displaying focal hypermetabolism in SWS relates to occipital lobe plasticity. Thus, when there is significant and early (likely intrauterine) occipital lobe injury resulting in severe hypometabolism and atrophy in the affected occipital lobe, there may be dramatic hypermetabolism in the contralateral intact occipital lobe (Figure 3), which appears normal on the MRI scan (11). This hypermetabolism seen in the intact hemisphere is not in any way related to epilepsy and should not be misinterpreted as a seizure focus. It is likely due to reorganizational changes (plasticity) in the intact occipital lobe, and is not specific to SturgeWeber syndrome but may also be seen in other conditions, such as focal cortical dysplasia affecting one occipital lobe (12).

\section{RETT SYNDROME}

Focal glucose hypermetabolism in interictal 18F-FDG PET studies has been reported in Rett syndrome, which is a neurodevelopmental disorder, affecting primarily girls, that progresses over several stages to severe intellectual and physical disabilities; the disorder results from mutations in the MECP2 gene [for a recent review see (13)]. Neuroimaging studies in Rett syndrome have revealed restriction of brain growth beginning in the first year of life; neuropathology examination suggests that axonodendritic connections are the most vulnerable in this neurodegenerative process. Naidu et al. (14) studied children with Rett syndrome using various neuroimaging approaches and found the largest changes in the frontal lobes, which showed hypoperfusion, as well as increased choline and reduced n-acetyl aspartate on MRS. However, 18F-FDG PET showed hypermetabolism in the frontal lobes not related to seizure activity (Figure 4). The authors concluded that the frontal cortex was hypermetabolic (rather than the occipital cortex hypometabolic) based on the SPECT hypoperfusion and MRS findings in frontal lobe of Rett patients. They hypothesized increased glutamate cycling in synapses leading to brain injury as the mechanism of frontal lobe hypermetabolism. The role of glutamate neurotoxicity in dendritic and synaptic injury is strongly supported by findings from mouse models of Rett syndrome (15). Indeed, the drug trofinetide, which is a glutamate antagonist, is currently undergoing clinical trials in children with Rett syndrome (16) and 18F-FDG PET may potentially serve as an objective biomarker to monitor therapeutic efficacy. 


\section{HYPOXIC-ISCHEMIC BRAIN INJURY IN THE NEWBORN}

Hypermetabolism in damaged brain regions can be seen in newborns in the acute phase following perinatal hypoxic-ischemic brain injury. Previous 18F-FDG PET studies in this population have not reported this phenomenon presumably because the babies were scanned in the subacute or chronic state. Instead, various patterns of hypometabolism were reported, extending beyond the affected structural abnormalities shown by CT scan (17). Subsequent studies in these babies suggested that persistently low cerebral glucose metabolic rates (i.e., quantitative CMRglc values) are associated with delayed development (18). Total CMRglc were found to be inversely correlated with the severity of the brain injury. Thus, neonates with the lowest CMRglc subsequently developed permanent neurological symptoms and cerebral palsy (19).

A transient hypermetabolism has been observed in the basal ganglia following the "neartotal' type of hypoxic-ischemic injury in the neonatal period, followed by severe hypometabolism in the lenticular nuclei and thalami when the child was restudied several years later (20) (Figure 5). This type of acute catastrophic injury may be seen in cases of placental abruption, uterine rupture or umbilical cord prolapse. The selective vulnerability of the basal ganglia in this setting may be related to the transient excess of glutamate receptors seen in the newborn basal ganglia compared to older children and adults (21). This form of injury is associated with the subsequent development of the dystonic (choreoathetoid, extrapyramidal) type of cerebral palsy as, indeed, was the sequela in this child. It was postulated that the transition from hypermetabolism to hypometabolism indicates the culmination of the brain injury due to excitotoxic mechanisms, which are mediated by glutamatergic neurotransmission [reviewed in (22)]. Unfortunately, the time course of the transient hypermetabolism is unknown and yet to be defined. Also unknown is whether transient hypermetabolism is seen in the 'watershed' types of neonatal hypoxic-ischemic brain injury (also referred to as the 'partial prolonged' type of brain injury) sometimes encountered at birth.

\section{INBORN ERRORS OF METABOLISM}

Transient hypermetabolism of the basal ganglia seen on 18F-FDG PET scans has been reported in several inborn errors of metabolism. This transient phase is followed by hypometabolism in the same brain region and indicates a selective vulnerability due to the abnormal biochemical state (i.e., metabolic 'toxins') in the specific disorder.

Proprionic acidemia is an organic acidemia associated with diverse neurological manifestations, including developmental delay, intellectual disability, seizures, optic neuropathy, and stroke-like episodes. The disorder is progressive and may also result in cardiac, gastrointestinal and renal complications. Serial MRI and 18F-FDG PET scans in 5 children with biochemically confirmed proprionic acidemia showed normal findings on both 18F-FDG PET and MRI in the early stages of the disorder. As the disease progressed, the 18F-FDG PET scans revealed glucose hypermetabolism in the basal ganglia and thalami with subsequent evolution to hypometabolism in the basal ganglia (Figure 6A,B,C), whereas MRI showed atrophy and abnormal signal in caudate and putamen with normal thalami (23). It was suggested by the investigators that the neurotoxins in this metabolic disorder have a predilection for the basal ganglia, activate glutamatergic mechanisms and eventually damage/destroy the basal ganglia. 
This notion is supported by the transient hypermetabolism in the basal ganglia in accordance with the dynamic nature of neurodegeneration during the course of this disorder.

A somewhat similar phenomenon has been reported in ethylmalonic aciduria, which is a severe organic aciduria affecting mitochondria and the vasculature. In addition to central nervous system involvement, ethylmalonic aciduria can also lead to changes in the retinal vessels, acrocyanosis, petechiae, and gastrointestinal symptoms. The central nervous system symptoms include hypotonia, physical and intellectual regression and, as the disease progresses, dystonia and spasticity. Neuroimaging studies in a 6-month-old boy with ethylmalonic aciduria showed a normal MRI scan but bilateral intense hypermetabolism in the caudate and putamen on 18F-FDG PET (Figure 6D,E). When imaged again a year later, 18F-FDG PET showed bilateral hypometabolism in the putamen, caudate head and frontal cortex, whereas MRI showed atrophy and infarcts in the basal ganglia (24). These findings are again consistent with the selective vulnerability of the basal ganglia in some inborn errors of metabolism.

18F-FDG PET scans have been performed on a number of other inborn errors of metabolism, often published in case reports. The general finding is hypometabolism but, in some cases, the hypometabolism is specific to, or most prominent in, the basal ganglia. These include glutaric aciduria type $1(25,26)$ and 3-methylglutaconic aciduria (27). It is unclear at which stage of disease progression these 18F-FDG PET studies were conducted. We postulate that had the 18F-FDG PET studies been performed at an earlier stage of rapid clinical regression in these disorders, the 18F-FDG PET scans might also have shown transient hypermetabolism in the basal ganglia. Furthermore, recently developed hybrid PET-MRI scanners provide a unique opportunity to determine the time course and relationship between structural, glucose metabolism and MR spectroscopic changes seen in this group of rare disorders.

\section{AUTOIMMUNE ENCEPHALITIS}

The autoimmune encephalitides comprise a group of disorders characterized by changes in mental status, seizures, dyskinesias or memory decline in association with the presence of serum and cerebrospinal fluid autoantibodies against brain proteins [for a review see (28)]. MRI scans in these disorders are often normal or may show increased fluid attenuated inversion recovery/T2 signal in various locations, for example, in one or both temporal lobes in limbic encephalitis. 18F-FDG PET scans may show focal hypermetabolism in various locations $(29,30)$.

Report of focal hypermetabolism in a case of autoimmune encephalitis involved a 15year-old boy with anti-N-methyl-D-aspartate receptor encephalitis whose brain MRI studies were repeatedly normal (29). On day 26 of admission, 18F-FDG PET showed global hypometobolism with a prominent focally intense hypermetabolic lesion in the right cerebellar cortex (Figure 7). The patient showed clinical signs of improvement after 2 courses of intravenous immunoglobulin therapy over 4 weeks. On day 46, repeat brain 18F-FDG PET showed overall improvement but in contrast to the previous $18 \mathrm{~F}-\mathrm{FDG}$ PET scan, the right cerebellar cortex showed focal hypometabolism. Focal hypermetabolism or more commonly hypometabolism in autoimmune encephalitis has been confirmed by other investigators and can be very useful in the early 
diagnosis in these disorders (30-32). In particular, some cases of suspected autoimmune encephalitis may be antibody-negative and 18F-FDG PET may contribute towards establishing a clinical diagnosis.

\section{CONCLUSION}

In this review, we have illustrated multiple examples of hypermetabolism that should not be interpreted as seizure activity on 18F-FDG PET scans. The radiologist or nuclear medicine physician interpreting these studies need to be aware that focal hypermetabolism does not always indicate a seizure focus and should be familiar with the less common causes of hypermetabolism. Since seizures do not emanate from the basal ganglia, the physician should also be aware that increased glucose metabolism in the basal ganglia may be a clue to an underlying metabolic disorder. In these cases, the timing of the 18F-FDG PET scan in relation to the course of a progressive neurological disorder should be noted and, if indicated, the study should be repeated to determine progressive changes in brain glucose metabolism.

Finally, when interpreting 18F-FDG PET scans of glucose metabolism visually (as in clinical studies), it is acknowledged that we are looking at 'relative' hypermetabolism since absolute quantification is not available and interpretation may be challenging. Some general rules may be helpful in this regard. For example, if supratentorial cortical metabolism is decreased, thalamic metabolism usually follows, caudate may follow, but not rest of the basal ganglia or brainstem. Frontal, parietal and temporal cortex metabolism are usually lower than metabolism in primary visual cortex (medial occipital cortex, calcarine cortex). Cerebellar metabolism is higher than most supratentorial cortical metabolism in the first few months postnatally, but never after the first year. This rule is particularly useful when deciding between supratentorial cortical hypometabolism versus basal ganglia hypermetabolism. Brainstem nuclei metabolism is usually around the same as that of the basal ganglia. Ultimately, the experience of the interpreting physician, as well as knowledge of the patient history, are crucial. 


\section{REFERENCES}

1. Chugani HT. Positron emission tomography in pediatric neurodegenerative disorders. Pediatr Neurol. 2019;100:12-25.

2. Schur S, Allen V, White A, et al. Significance of FDG-PET hypermetabolism in children with intractable focal epilepsy. Pediatr Neurosurg 2018;53:153-162.

3. Chugani HT, Shewmon DA, Khanna S, Phelps ME. Interictal and postictal focal hypermetabolism on positron emission tomography. Pediatr Neurol 1993;9:10-15.

4. Ackermann RF, Chugani HT, Handforth A, Moshe S, Caldecott-Hazard S, Engel J. Autoradiographic studies of cerebral metabolism and blood flow in rat amygdala kindling. In: Wada JA, ed. Kindling 3. New York, NY: Raven Press; 1986:73-87.

5. Chugani HT, Mazziotta JC, Phelps ME. Sturge-Weber syndrome: a study of cerebral glucose utilization with positron emission tomography. J Pediatr 1989;114:244-253.

6. Juhász C, Batista CE, Chugani DC, Muzik O, Chugani HT. Evolution of cortical metabolic abnormalities and their clinical correlates in Sturge-Weber syndrome. Eur $J$ Paediatr Neurol 2007;11:277-284.

7. Pinton F, Chiron C, Enjolras O, Motte J, Syrota A, Dulac O. Early single photon emission computed tomography in Sturge-Weber syndrome. J Neurol Neurosurg Psychiatry 1997;63:616-621.

8. Jacoby CG, Yuh WT, Afifi AK, Bell WE, Schelper RL, Sato Y. Accelerated myelination in early Sturge-Weber syndrome demonstrated by MR imaging. J Comput Assist Tomogr 1987;11:226-231.

9. Juhász C, Hu J, Xuan Y, Chugani HT. Imaging increased glutamate in children with Sturge-Weber syndrome: association with epilepsy severity. Epilepsy Res 2016;122:6672 .

10. Alkonyi B, Chugani HT, Juhász C. Transient focal cortical increase of interictal glucose metabolism in Sturge-Weber syndrome: implications for epileptogenesis. Epilepsia 2011;52:1265-1272.

11. Batista CE, Juhász C, Muzik O, Chugani DC, Chugani HT. Increased visual cortex glucose metabolism contralateral to angioma in children with Sturge-Weber syndrome. Dev Med Child Neurol 2007;49:567-573.

12. Jeong JW, Tiwari VN, Shin J, Chugani HT, Juhász C. Assessment of brain damage and plasticity in the visual system due to early occipital lesion: comparison of FDG-PET with diffusion MRI tractography. J Magn Reson Imaging 2015;41:431-438. 
13. Gold WA, Krishnarajy R, Ellaway C, Christodoulou J. Rett syndrome: a genetic update and clinical review focusing on comorbidities. ACS Chem Neurosci 2018;9:167-176.

14. Naidu S, Kaufmann WE, Abrams MT, et al. Neuroimaging studies in Rett syndrome. Brain Dev 2001;suppl 1:S62-71.

15. Maezawa I, Jin LW. Rett syndrome microglia damage dendrites and synapses by the elevated release of glutamate. J Neurosci 2010;30:5346-5356.

16. Glaze DG, Neul JL, Kaufmann WE, et al. Double-blind, randomized, placebo-controlled study of trofinetide in pediatric Rett syndrome. Neurology 2019;92:e1912-e1925.

17. Doyle LW, Nahmias C, Firnau G, Kenyon DB, Garnett ES, Sinclair JC. Regional cerebral glucose metabolism of newborn infants measured by positron emission tomography. Dev Med Child Neurol 1983;25:143-151.

18. Suhonen-Polvi H, Kero P, Korvenranta H, et al. Repeated fluorodeoxyglucose positron emission tomography of the brain in infants with suspected hypoxic-ischaemic brain injury. Eur J Nucl Med 1993;20:759-765.

19. Thorngren-Jerneck K, Ohlsson T, Sandell A, et al. Cerebral glucose metabolism measured by positron emission tomography in term newborn infants with hypoxic ischemic encephalopathy. Pediatr Res 2001;49:495-501.

20. Batista CE, Chugani HT, Juhász C, Behen ME, Shankaran S. Transient hypermetabolism of the basal ganglia following perinatal hypoxia. Pediatr Neurol 2007;36:330-333.

21. Greenamyre T, Penney JB, Young AB, Hudson C, Silverstein FS, Johnston MV. Evidence for transient perinatal glutamatergic innervation of globus pallidus. $J$ Neurosci 1987;7:1022-1030.

22. Johnston MV. Excitotoxicity in neonatal hypoxia. Ment Retard Dev Disabil Res Rev 2001;7:229-234.

23. Al-Essa M, Bakheet S, Patay Z, et al. 18Fluoro-2-deoxyglucose (18FDG) PET scan of the brain in propionic acidemia: clinical and MRI correlations. Brain Dev 1999;21:312317.

24. Al-Essa MA, Al-Shamsan LA, Ozand PT. Clinical and brain 18fluoro-2-deoxyglucose positron emission tomographic findings in ethylmalonic aciduria, a progressive neurometabolic disease. Eur J Paediatr Neurol 1999;3:125-127.

25. Al-Essa M, Bakheet S, Patay Z, et al. Fluoro-2-deoxyglucose (18FDG) PET scan of the brain in glutaric aciduria type 1: clinical and MRI correlations. Brain Dev 1998;20:295301. 
26. Awaad Y, Shamoto H, Chugani H. Hemidystonia improved by baclofen and PET scan findings in a patient with glutaric aciduria type I. J Child Neurol 1996;11:167-169.

27. Al-Essa M, Bakheet S, Al-Shamsan L, Patay Z, Powe J, Ozand PT. 18Fluoro-2deoxyglucose (18FDG) PET scan of the brain in type IV 3-methylglutaconic aciduria: clinical and MRI correlations. Brain Dev 1999;21:24-29.

28. Graus F, Titulaer MJ, Balu R, et al. A clinical approach to diagnosis of autoimmune encephalitis. Lancet Neurol 2016;15:391-404.

29. Maqbool M, Oleska DA, Huq AH, Salman BA, Khodabakhsh K, Chugani HT. Novel FDG-PET findings in anti-NMDA receptor encephalitis: a case based report. $J$ Child Neurol 2011;26:1325-1328.

30. Newey CR, Sarwal A, Hantus S. [18F]-fluoro-deoxy-glucose positron emission tomography scan should be obtained early in cases of autoimmune encephalitis. Autoimmune Dis 2016;9450452.

31. Solnes LB, Jones KM, Rowe SP, et al. Diagnostic value of 18F-FDG PET/CT versus MRI in the setting of antibody-specific autoimmune encephalitis. $J$ Nucl Med 2017;58:1307-1313.

32. Tripathi M, Tripathi M, Roy SG, et al. Metabolic topography of autoimmune nonparaneoplastic encephalitis. Neuroradiology 2018;60:189-198. 

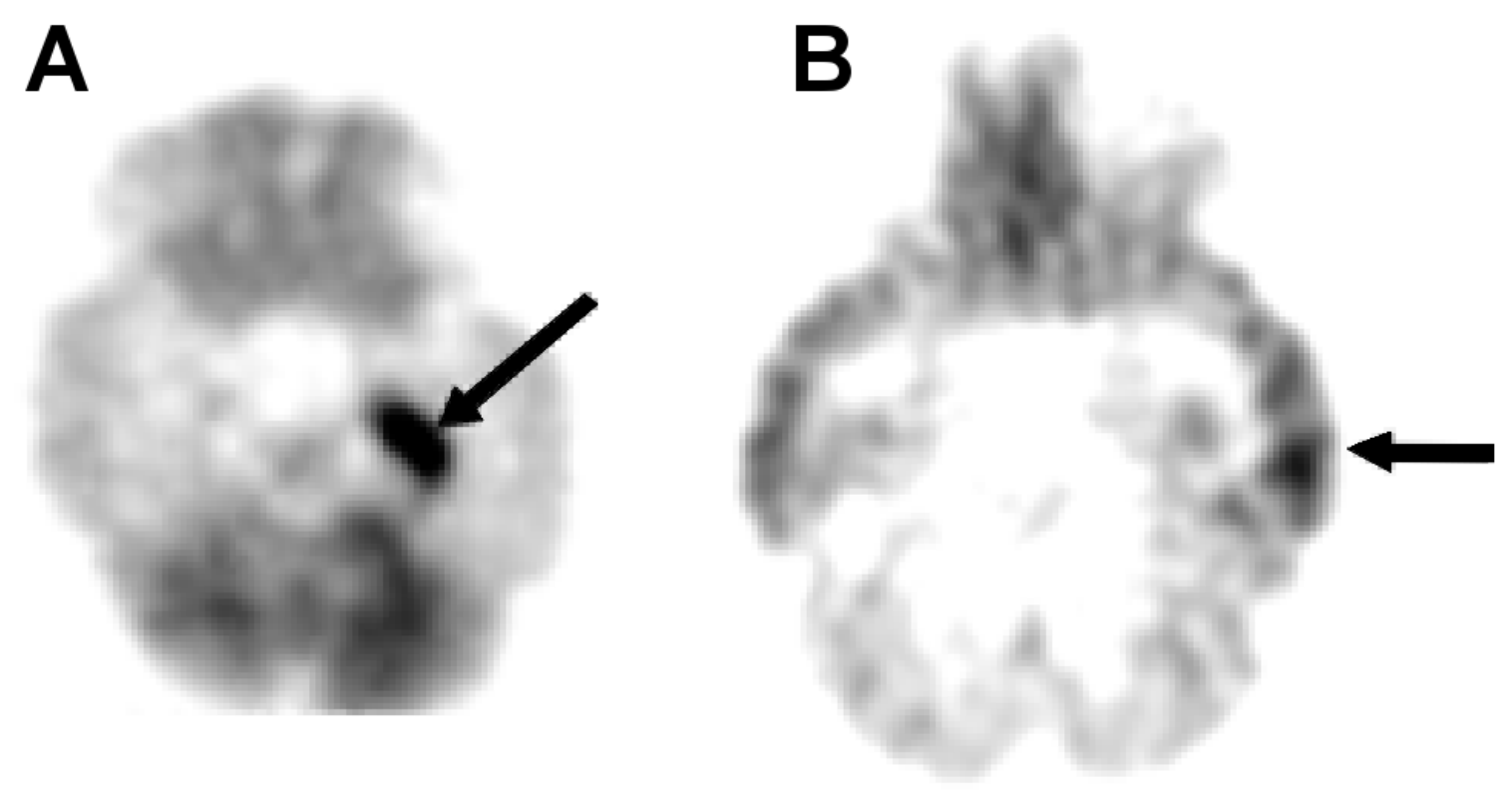

Figure 1. (A) Ictal 18F-FDG PET scan in a 14 month old child with infantile spasms who fortuitously developed a convulsive seizure 12 minutes after administration of 18F-FDG. Note the intense hypermetabolism (arrow) in the left medial temporal lobe; (B) Interictal hypermetabolism in an adolescent with left temporal lobe epilepsy who showed spiking on the electroencephalogram during the entire $18 \mathrm{~F}-\mathrm{FDG}$ uptake period, producing left temporal cortex hypermetabolism (arrow). No actual seizures were witnessed clinically or observed on the electroencephalogram. Without electroencephalography, this 18F-FDG PET study could have been interpreted as right temporal cortex hypometabolism, thus falsely lateralizing the seizure focus 


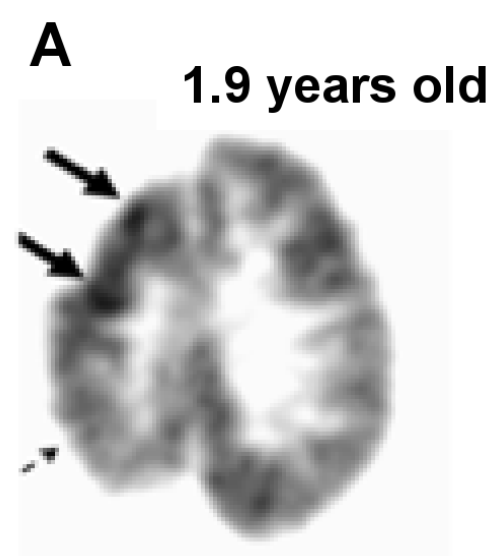

\section{B}

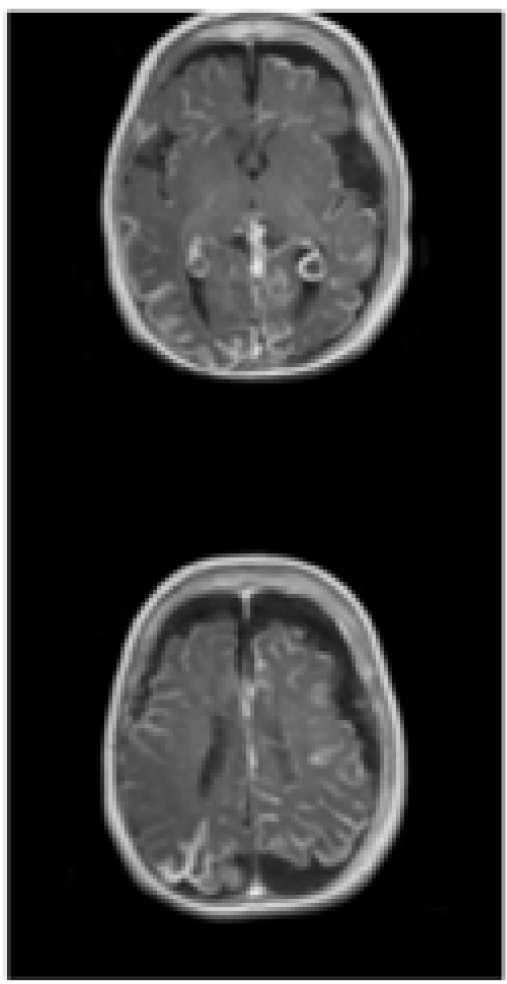

C
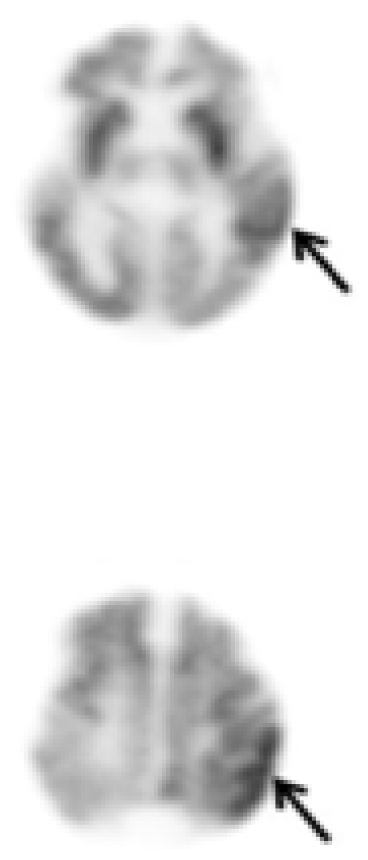

$\mathbf{R}$

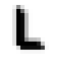

Figure 2. (A) Transient focal hypermetabolism evolving to hypometabolism and brain injury in Sturge-Weber syndrome may undergo a different temporal course in various regions within the affected hemisphere. In this child, 18F-FDG PET scan showed interictal right frontal cortex hypermetabolism at age 1.9 years evolving to hypometabolism at age 2.7 years (solid arrows), whereas right parietal cortex hypometabolism was already present at age 1.9 years and unchanged at 2.7 years (broken arrows); (B) and (C) MRI and 18F-FDG PET in a child with bilateral brain involvement showing both hypometabolism and hypermetabolism (arrows). 

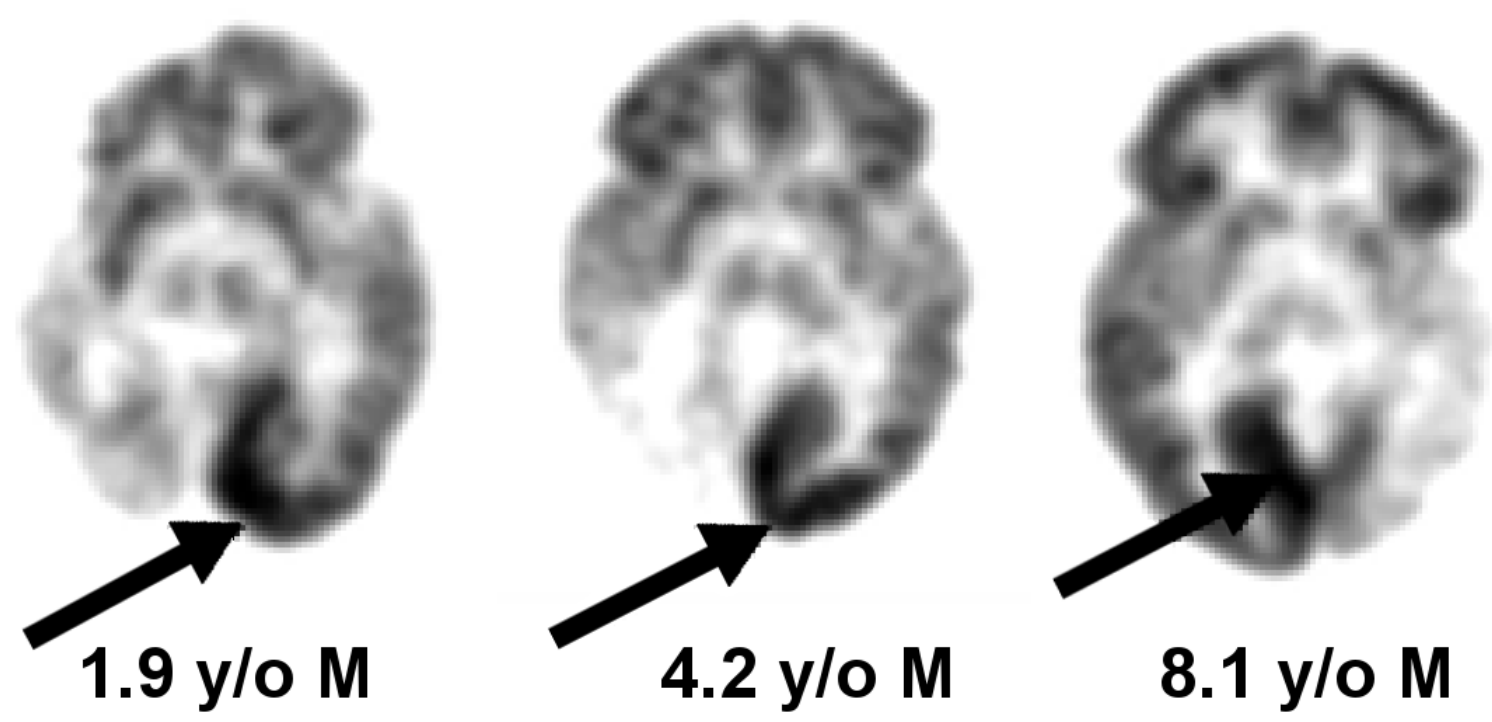

Figure 3. 18F-FDG PET scans of 3 children with unilateral leptomeningeal angiomatosis in Sturge-Weber syndrome. The arrows point to the normal occipital cortex showing hypermetabolism, as compared to the contralateral affected hemisphere which shows severe hypometabolism and atrophy. 


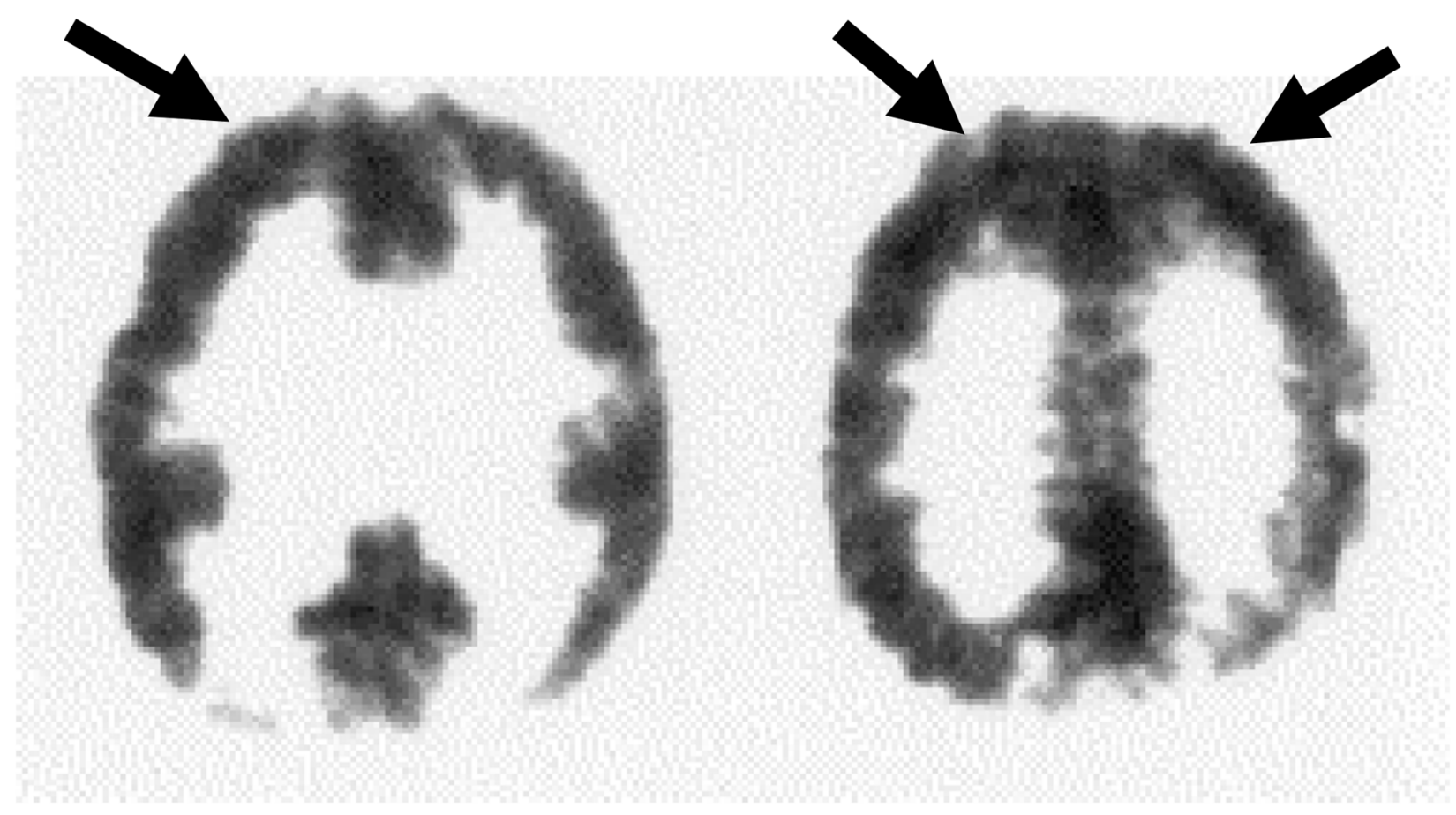

Figure 4. Eight year old girl with Rett syndrome whose 18F-FDG PET scan shows bilateral frontal cortex hypermetabolism (arrows). 


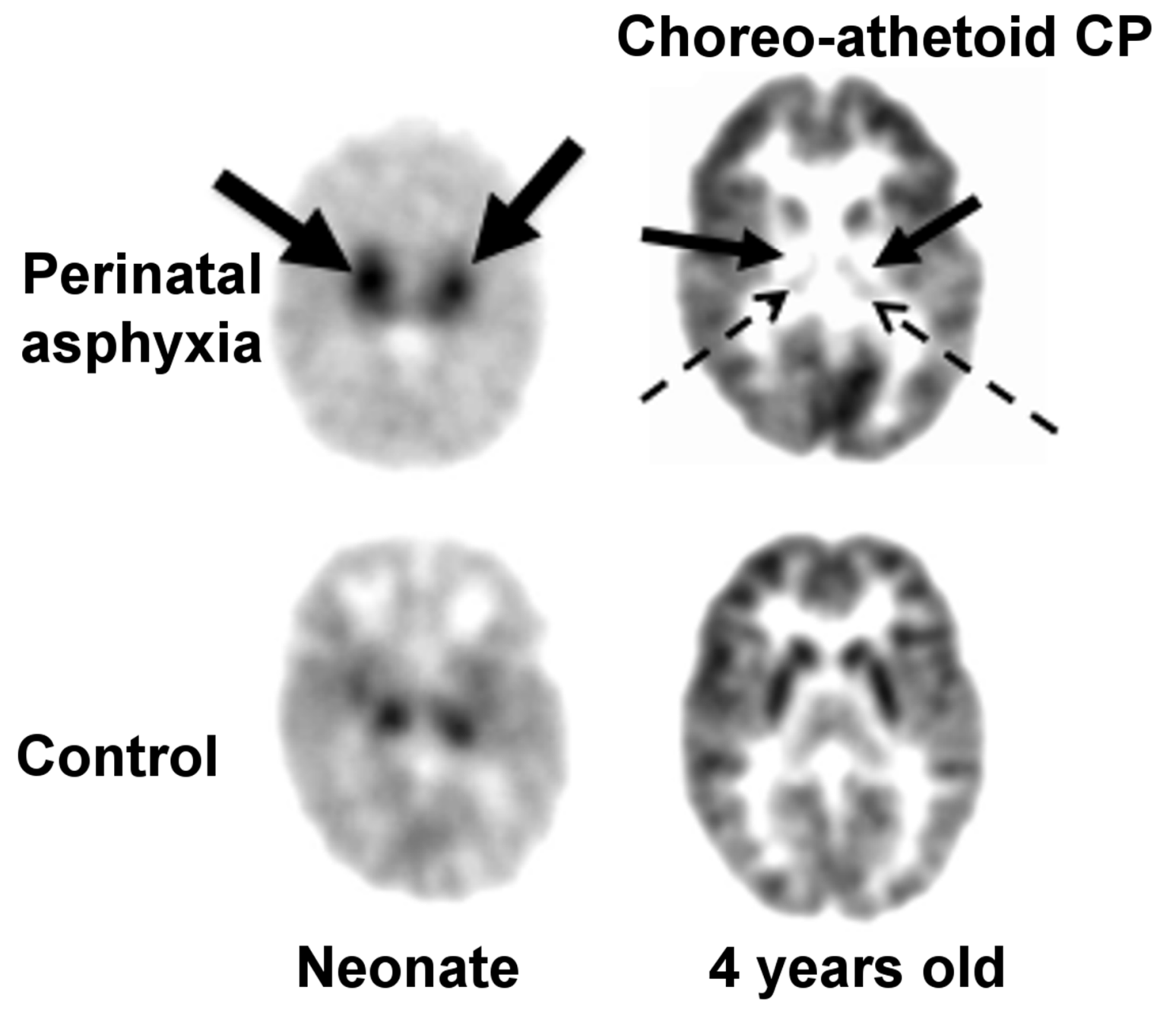

Figure 5. 18F-FDG PET scan of a child who suffered hypoxic ischemic encephalopathy at birth and later developed dystonic/choreoathetoid cerebral palsy. The left image from the newborn period showed intense hypermetabolism in the basal ganglia (solid arrows) compared to control. The right image at 5 years of age showed severe hypometabolism in the lenticular nuclei (solid arrows) and thalami (dotted arrows). Note the relative preservation of metabolism in the cerebral cortex. 


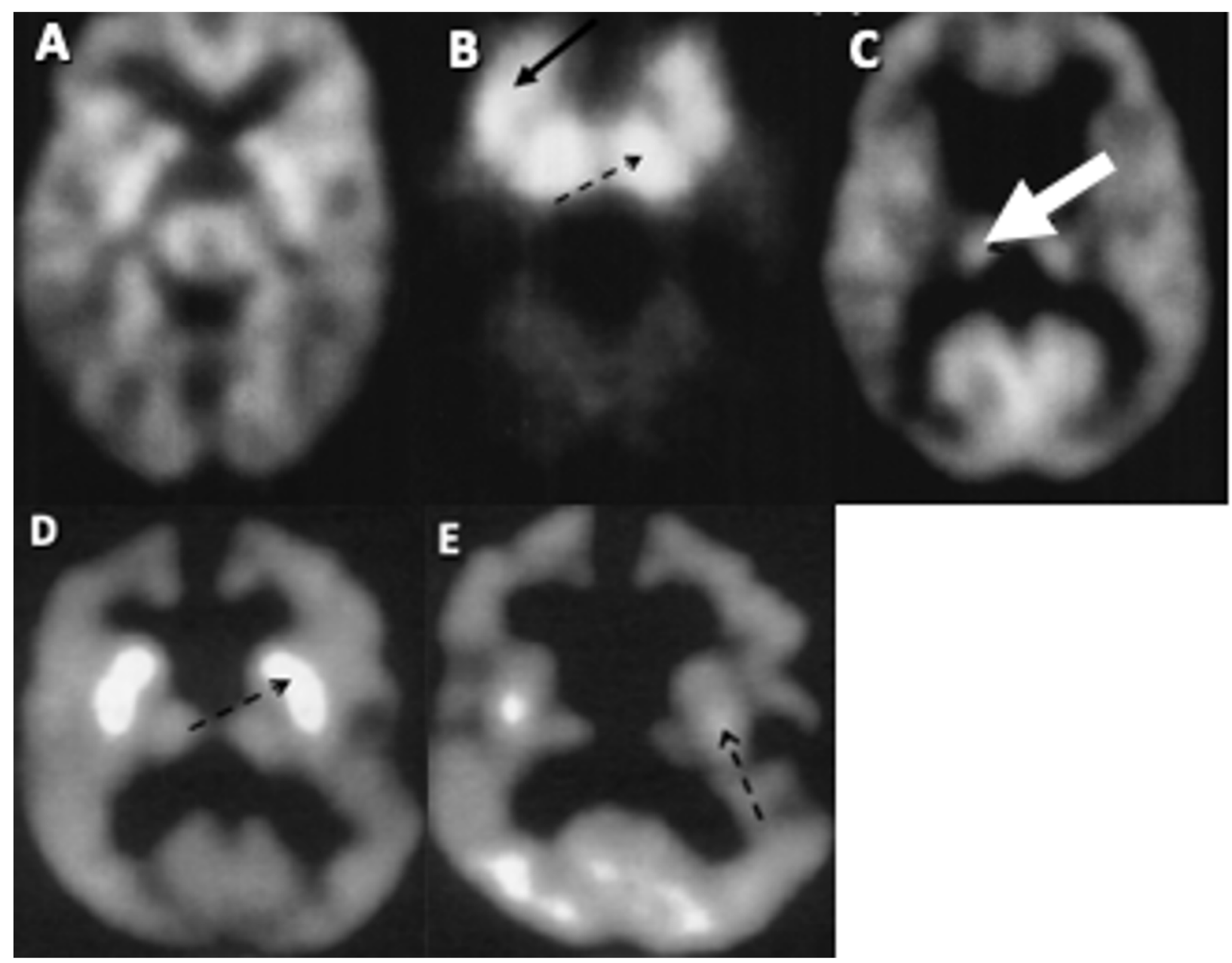

Figure 6. (A,B,C) 18F-FDG PET scans of 3 children with proprionic acidemia at different stages of the disease. (A) At age 1 month, this baby showed a relatively normal pattern of glucose metabolism for age; (B) this 2 months old infant showed intense hypermetabolism in the basal ganglia (solid arrow) and thalamus (dashed arrow); (C) this 2 year old child showed relatively normal glucose metabolism in the thalamus (dashed arrow), but the basal ganglia showed absent metabolic activity; (D,E) 18F-FDG PET images in a boy with ethylmalonic aciduria. (D) At 2 years of age, intense bilateral hypermetabolism is seen in the caudate nucleus and putamen (dashed arrow); (E) when scanned again at age 3 years, there has been evolution of the hypermetabolism to hypometabolism (dashed arrow). 

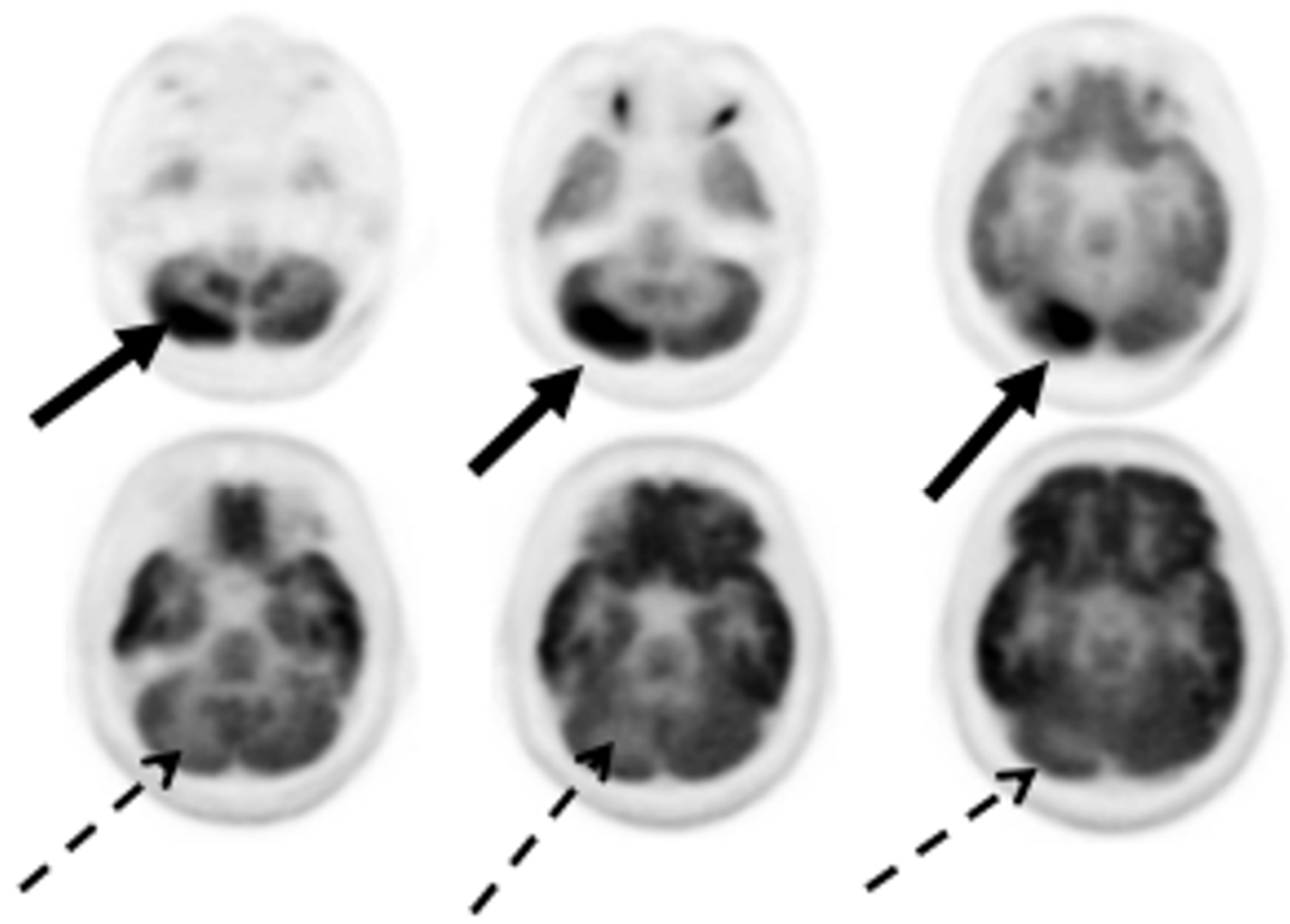

Figure 7. 18F-FDG PET scans from a 15-year-old boy with anti-N-methyl-D-aspartate receptor encephalitis. Top row: focal intense hypermetabolism in right cerebellar cortex (solid arrows) and diffuse hypometabolism of bilateral cerebral hemispheres. Bottom row: subsequent focal hypometabolism in the right cerebellar cortex (dashed arrows), corresponding with the initially hypermetabolic region. 